\title{
Spatio-chromatic ICA of a Mosaiced Color Image
}

\author{
David Alleysson ${ }^{1}$ and Sabine Süsstrunk ${ }^{2}$ \\ 1 Laboratory for Psychology and NeuroCognition, CNRS UMR 5105 \\ Université Pierre-Mendès France, Grenoble, France \\ David.Alleysson@upmf-grenoble.fr \\ 2 Audiovisual Communications Laboratory \\ École Polytechnique Fédérale de Lausanne, Switzerland \\ Sabine.Susstrunk@epfl.ch
}

\begin{abstract}
We analyze whether Independant Component Analysis (ICA) is an appropriate tool for estimating spatial information in spatiochromatic mosaiced color images. In previous studies, ICA analysis of natural color scenes (Hoyer et al. 2000; Tailor et al., 2000; Wachtler et al., 2001; Lee et al. 2002) have shown the emergence of achromatic patterns that can be used for luminance estimation. However, these analysis are based on fully defined spatio-chromatic images, i.e. three or more chromatic values per pixel. In case of a reduced spatio-chromatic set with a single chromatic measure per pixel, such as present in the retina or in CFA images, we found that ICA is not an appropriate tool for estimating spatial information. By extension, we discuss that the relationship between natural image statistics and the visual system does not remain valid if we take into account the spatio-chromatic sampling by cone photoreceptors.
\end{abstract}

\section{Introduction}

The statistical analysis of natural scenes, as viewed by human observers, has given new insight into the processing and functionality of the human visual system. Pioneer work by Field (1987) and Barlow (1989) has established the relation between redundancy reduction in natural scenes and the visual system's receptive fields. Using gray-scale natural scene imagery, Olshausen \& Field (1996) show that representing images with sparse (less redundant) code leads to spatial basis functions that are oriented, localized, and band-pass, and resemble the receptive field structures of the primary cortex cells. Bell \& Sejnowski (1997) found that sparseness could be appropriately formalized using Independent Component Analysis (ICA), and show that independent components of natural scenes act as edge filters.

For the case of color, Buchsbaum \& Gottschalk (1983) use Principal Component Analysis (PCA) of L, M, and S cone signals to derive post-receptoral mechanisms: luminance and opponent chromatic channels (blue minus yellow, and red minus green). They propose that this de-correlated coding reduces the 
information transmitted to the optical nerve. Later, Attick \& Redlich (1992) formalized the relation between natural color scenes and retinal functions. They show that a retinal filter is consistent with a whitening process of the natural scene structure when noise is taken into account.

Finally, the use of hyperspectral images to simulate cone responses has allowed to precisely analyze the spatio-chromatic structure of natural scenes and confirmed previous studies (Wachtler et al., 2001). Ruderman et al. (1998) show that the principal components of natural color images, as sampled by cones, are consistent with post-receptoral receptive fields and provide reduced signals. Using ICA, Hoyer et al. (2000), Tailor et al. (2000) and Lee et al. (2002) found that natural color image statistics could account for simple and complex color opponent receptive fields in the primary cortex.

From these studies, it seems that the post-receptoral mechanisms of the human visual system correspond to a statistical analysis of natural scenes and provide a redundancy reduction. But none of these studies take into account that cone sampling already results in a reduced spatio-chromatic signal. In the retina, the three types of cones (L, M and S) form a mosaic such that only a single chromatic sensitivity is sampled at each spatial location. Thus, the spatio-chromatic signals are already reduced by a factor of three compared to fully defined spatio-chromatic signals of a natural scene (or color image). Doi et al. (2003) did propose a study where the cone mosaic is taken into account. They used a local arrangement of cones (127), from which they sampled LMS responses to construct vectors and perform ICA analysis. Although this method gives interesting results, it is still not realistic for simulating cone sampling since only a small part of the entire mosaic is used. Their study actually corresponds to analyzing the signal of a part of the retina scanning a natural scene.

In a previous paper (Alleysson \& Süsstrunk, 2004a), we studied whether we can find a similar correspondence with the processing of the human visual system by statistical analysis of natural color images sampled with a spatiochromatic mosaic. In that preliminary study, we have restricted our analysis to Principal Component Analysis (PCA), a second order statistical analysis that performs a simple de-correlation of a signal. We used RGB color images instead of LMS images constructed from hyperspectral data, and we assumed a regular arrangement of RGB samples instead of a random arrangement, such as given by the cone distribution in the retina. Actually, this experimental set-up coincides with many digital camera sensors, since most are single-chip and use a Color Filter Array (CFA) to provide color responses. Such systems sample a single chromatic sensitivity per pixel and need to interpolate the missing information to render color images (Alleysson et al., 2002). We then investigated if a spatiochromatic analysis using PCA is able to help the reconstruction of the spatial information.

The conclusion of the previous study is that spatial information cannot be recovered with a second order statistical analysis. In this paper, we extend our approach and study if a higher order statistical analysis, given by Independant Component Analysis, provides a method to separate spatial information from 
chromatic information in a spatio-chromatic mosaiced color image. If such an approch is successfull, it would provide insights into the human visual processing and help design better demosaicing algorithms for CFA images.

\section{Spatio-chromatic ICA of Color Images}

Independent Component Analysis is a high order statistical analysis, which supposes that the signals measured by a sensor (observations) are an unknown linear mixing of unknown independent sources. Rather than exploiting the correlation between observations, as is done with Principal Component Analysis, high order statistics are used in ICA.

Suppose $N$ measures of $T$ random variables $x_{i j}(i \in[1 . . N], j \in[1 . . T])$. ICA presumes that these observations come from an instantaneous linear mixture (i.e. verified for all $i$ ), given by $\mathbf{A}$ of $T$ independent sources $\mathbf{s}_{j}$, as follows:

$$
\mathbf{x}_{j}=\mathbf{s}_{j} \mathbf{A}
$$

Here we consider only the case where the number of sources is equal to the number of observations. ICA analysis will try to find $T$ independent vectors $\mathbf{u}_{j}$, which are representatives of the $T$ vectors $\mathbf{x}_{j}$ through a linear transformation B: $\mathbf{u}_{j}=\mathbf{x}_{j} \mathbf{B}$ for all $i$. The estimated vectors $\mathbf{u}_{j}$ are independent when their probability distributions $f_{\mathbf{u}}$ factorize in $f_{\mathbf{u}}(\mathbf{u})=\Pi_{j} f_{\mathbf{u}_{j}}\left(\mathbf{u}_{j}\right)$ or, equivalently, when there is zero mutual information between them: $I\left(\mathbf{u}_{j}, \mathbf{u}_{k}\right)=0$ (Bell \& Sejnowski, 1997).

In this paper, we have used a freeware Matlab Toolbox called FastICA (Hyvärinen, 2004) for the ICA analysis, which uses an approximation of the negative normalized entropy (negentropy) as a criterion for independance, and is equivalent to a fixed point infomax or maximum likelihood approach (Hyvärinen \& Oja, 2000).

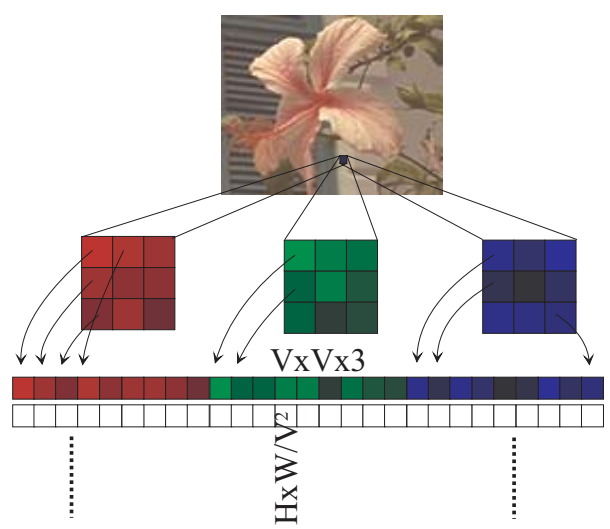

Fig. 1. Decomposition of the original image into a spatio-chromatic vector 
Given an image $\mathbf{I}_{i, j, c}$, defined by a three-dimensional matrix of size $H \times W \times 3$, we can construct a two-dimensional matrix $\mathbf{X}$ that contains for each row a vector $\mathbf{x}_{j}$ composed of spatial neighbors of size $V$ of a pixel for all three color layers (see Figure 1). In our previous paper (Alleysson \& Süsstrunk, 2004a), we used the neigborhood of each pixel. In this paper, we reduced the matrix size by using only the neigborhood of a pixel separated by the interval of $V$. The estimation of the unmixing matrix $\mathbf{B}$ gives the same result in both conditions, certainly because of the stationarity of spatial variables in images. Thus, the size of $\mathbf{X}$ is $\left(H W / V^{2}\right) \times\left(3 V^{2}\right)$. This matrix, on which we can apply an ICA, can be interpreted as containing on each row a representation of the spatio-chromatic random variables of a color image.

Once the matrix $\mathbf{X}$ is constructed from the image, the ICA analysis is performed. The resulting matrices $\mathbf{U}_{e}, \mathbf{A}_{e}, \mathbf{B}_{e}$ are the estimated sources $\left[\mathbf{u}_{j}\right]$ for all $i$, mixing, and unmixing matrices, respectively. The repesentation of estimated sources is not very usefull. Sources are estimated through a permutation and scaling indetermination (Hyrävinen \& Oja, 2000), which could modify the role and gain of color, horizontal, and vertical variables in the spatio-chromatic estimated sources. But the column $\mathbf{a}_{i}$ of $\mathbf{A}_{e}$ represent the basis functions that are applied to the sources to form the observations. In Figure 2, these functions are represented for a $3 \times 3$ neigborhood in the original image. They are ordered in decreasing order, according to $\sum_{i}\left|a_{i j}\right|$. Each function is recast on a $3 \times 3 \times 3$ arrangement and is represented as a color patch rescaled between $[0,1]$.
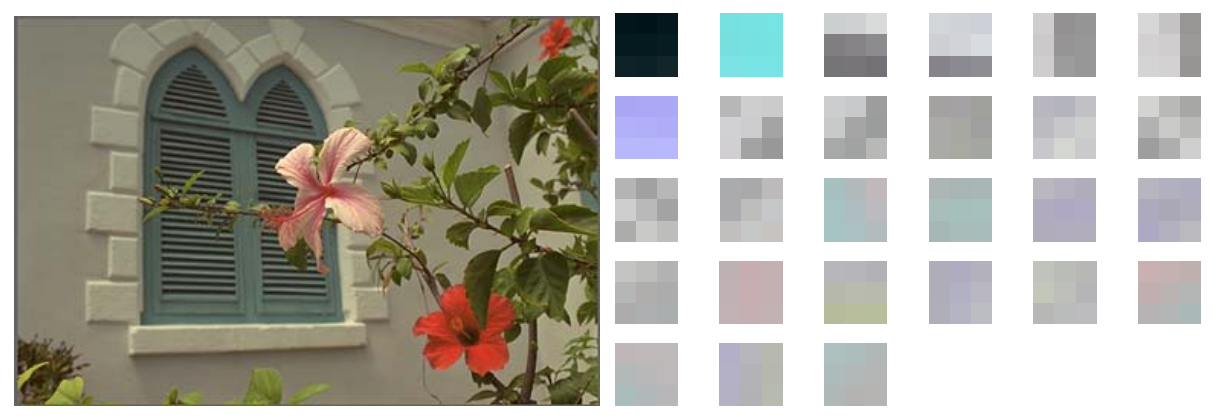

Fig. 2. Basis functions of the ICA of the color image ordered according to $\sum_{i}\left|a_{i j}\right|$

It is possible to partially reconstruct the image using only a few basis functions. This can be achieved by exchanging the entries of the undesired column vectors in $\mathbf{A}_{e}$ with zero. Call $\mathbf{A}_{1}$ the matrix $\mathbf{A}_{e}$ in which some column vectors have been replaced with zeros. The partially reconstructed two-dimensional image matrix is then given by $\mathbf{X}_{1}=\mathbf{U}_{e} \mathbf{A}_{1}$ from which we can reconstruct an image. As an example, Figure 3 shows reconstruction examples with the first, second, third, fourth, and seventh basis functions, as well as the reconstruction with first through seventh components. 

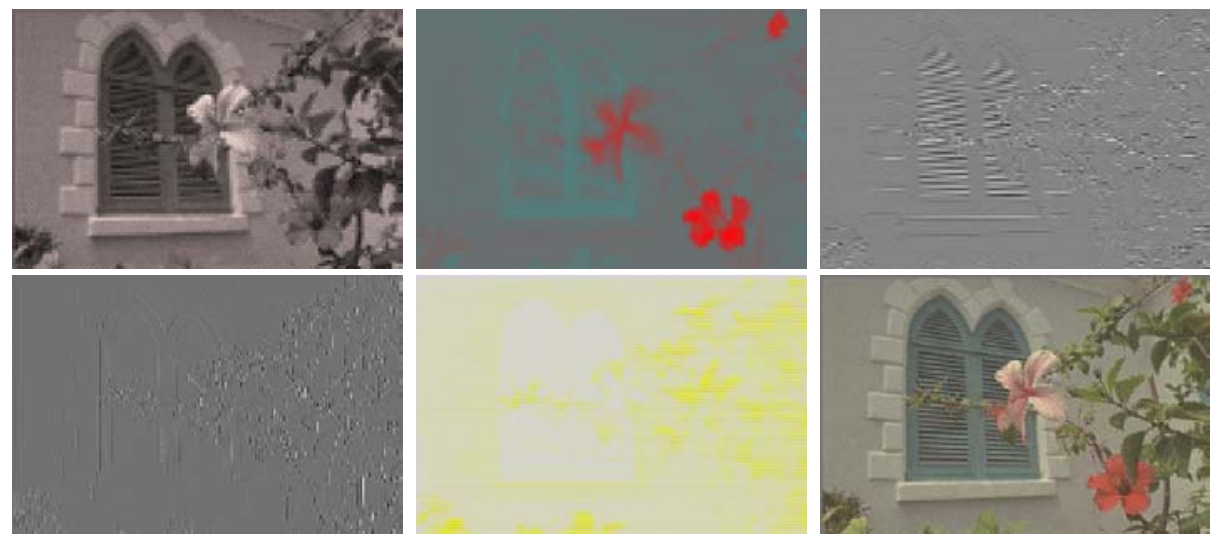

Fig. 3. Partial reconstruction of the original image (Figure 2) using only a few basis functions. (Top) Using the first, the second, and the third basis function. (Bottom) The reconstruction using the fifth, the seventh, and using first through seventh basis functions

The results of Figures 2 and 3 are compatible with pevious results in spatiochromatic ICA (Hoyer et al. 2000, Wachtler et al., 2001; Tailor et al. 2000), where it was found that basis functions are composed of achromatic band-pass oriented functions and red/green and blue/yellow basis functions. We can see in Figure 2 that several basis functions are achromatic and do not carry chromatic information, but a part of the spatial information. This is even better illustrated in the partial image reconstruction. For example, the reconstruction with only the first component (top-left of Figure 3 ) is mainly achromatic with coarse luminance. Also, the reconstruction with only the third and fourth basis functions contain horizontal, respectively vertical achromatic information of the original image. Incidentally, all achromatic basis functions in Figure 2 carry part of spatial information of the original image, and the chromatic basis functions are composed of red/green and blue/yellow components.

\section{Spatio-chromatic ICA of a Mosaiced Images}

Retinal images and images captured with single-chip digital cameras with a Color Filter Array (CFA) have only a single chromatic value per spatial location. Figure 4 illustrates a mosaiced image according to the Bayer CFA. It has been shown that such image can be decomposed into a sum of an achromatic image with full spatial resolution plus a subsampled chromatic image (Alleysson et al. 2002). The sub-sampling of chromatic information at the sensor level does not affect luminance but only chrominance. Moreover, luminance and chrominance have localized energies in the Fourier domain, allowing their estimation by frequency selection. A full color image is then obtain by interpolating chrominance and add it to the estimated luminance. In many cases, however, luminance and 
chrominance alias in the Fourier domain when their representations occupy too large of a region in the frequency spectrum and overlap (Alleysson \& Süsstrunk, 2004b). In that case, the frequency selection algorithm cannot separate the two. Thus, one can investigate if a statistical method allows separating luminance and chrominance in a mosaiced image. Moereover, a linear decomposition method should work because the luminance and chrominance are composed linearly in a mosaiced image.

Since there are achromatic basis functions in an ICA decomposition of a color image, we can hope that there are also achromatic functions in an ICA decompostion of a mosaiced image. In that case, it should be possible to use only these achromatic functions to reconstruct the luminance part of a mosaiced image, and to improve existing demosaicing algorithms.

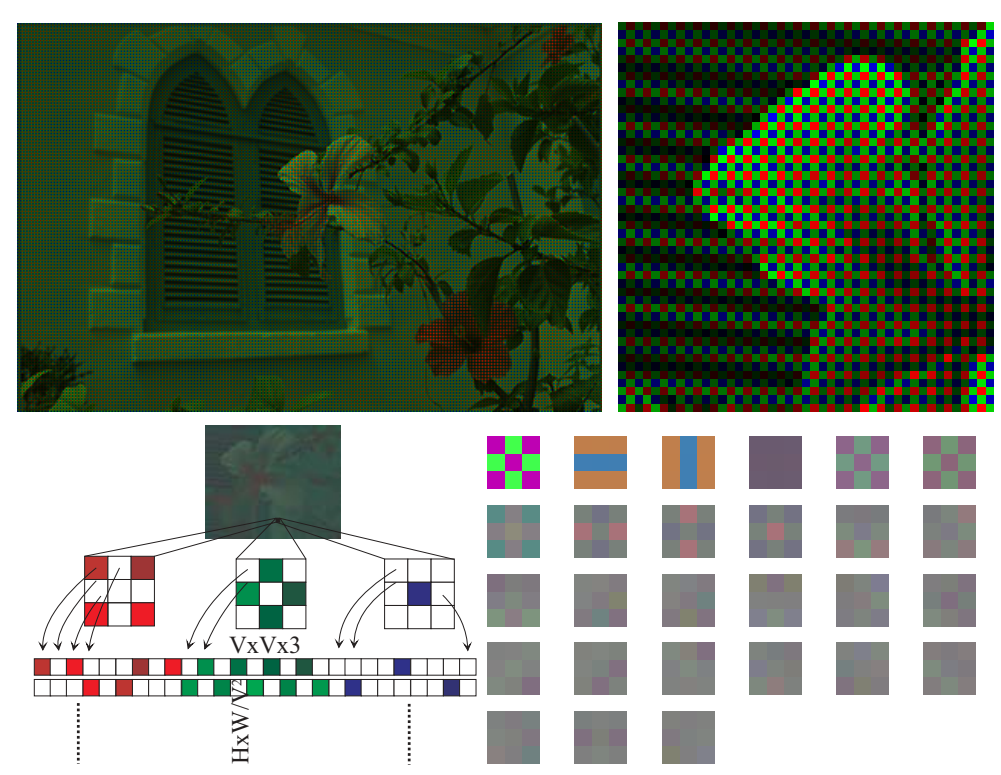

Fig. 4. (Top) A mosaiced image according to the Bayer CFA. The image appears green as there are twice as many green pixels as red and blue pixels. (Bottom) The de-composition of a mosaiced image into a two-dimensional matrix and the resulting basis functions of the ICA decomposition

It could be seen in Figure 4 that none of the basis function have an achromatic characteristic. This mean that, like the PCA, ICA is not a good tool for estimating the achromatic information in a mosaiced color image.

\section{Conclusion}

Principal and Independent Component Analysis are not adequate methods to estimate statistically the luminance information in a mosaiced color image. Possible reasons for this failure could be the difference in spatial resolution of the 
luminance and chromatic signal in a mosaiced image that is not taken into account by using a spatio-chromatic decomposition based on a fixed neigborhood size. Also, even if the luminance and chrominance additively compose a mosaiced image, it is possible that in case of aliasing between them, a non-linear method should be used. Finally, FastICA provides a way to obtain independent signals from mixed ones, but there may be other ICA methods that do converge to an independence between achromatic and chromatic signals.

With regards to the visual system, the relationship between statistical analysis of natural scenes and the visual system does not remain clear when considering the first step of neural activity in the visual system, i.e. the sampling of visual information by cones. It was quite intuitive that the decorrelation process of the PCA described in the previous paper (Alleysson \& Süsstrunk, 2004a) would not be able to separate spatial and chromatic information in spatio-chromatic samples. For example, in the schema proposed by Bell \& Sejnowski (1997), decorrelation is the first step of visual processing and is part of retinal processing. There is a correspondance between retinal receptive fields and a decorrelation process. Atick \& Redlich 1992 also favor the hypothesis of decorrelation by retinal functions. However, it is known that the separation of luminance and chominance does not completly arise in the retina, because there are pathways at the output of the retina that contain multiplexed spatial and chromatic signals. But it is less intuitive that an independant decomposition is still not able to perform such a separation, as this analysis is supposedly happening in the brain, at least at the primary cortex level.

Thus, there are many new interesting venues to study to reconcile the statistical analysis of natural scenes with the visual system's functionality, taking into account the real spatio-chromatic sampling by the cones. One is to find if the separation of spatial and chromatic information could arise after the primary cortex level by some other kind of statistical analysis. Another is to find if the processing of the reduced spatio-chromatic signals in the retina and primary cortex could be equivalent to a statistical analysis of a complete, fully populated chromatic signal. In that case, the visual system could give insights for statistical analysis of natural scenes based on reduced information. For example, a mosaiced image contains already a kind of decorrelated information because of subsampling (if neighboring pixels have high correlation, their correlation decreases after subsampling). It is therefore plausible that the retino-cortical projection provides an independent transformation, without having recourse to a complicated and iterative process.

\section{References}

Alleysson, D., Süsstrunk, S., Hérault, J. Color demosaicing by estimating luminance and opponent chromatic signals in Fourier domain. Proc. IS\&T/SID 10th Color Imaging Conference, Scottsdale, 2002, 331-336.

Alleysson, D., Süsstrunk, S., Spatio-chromatic PCA analysis of a mosaiced image. Proc. IS\&T 2nd European Conf. on Color in Graphics, Image and Vision (CGIV'04). Aachen, Germany, 2004a, 311-314. 
Alleysson, D., Süsstrunk, S., Aliasing in digital cameras. SPIE Electronic Imaging Newsletter, Special Issue on Smart Image Acquisition and Processing, 14(1), 2004b, 1.

Atick J.J., Redlich A.N., What does the retina know about natural scenes? Neural Computation 4, 1992, 196-210.

Barlow H.B., Unsupervised learning. Neural Computation 1, 1989, 295-311.

Bauchsbaum G., Gottschalk A., Trichromacy, opponent colours coding and optimum colour information in the retina. Proc. R. Soc. Lond. B220, 1983, 89-113.

Bell A.J., Sejnowski T.J., The independent components of natural scenes are edge filters. Vis. Res., 1997, 3327-3338.

Doi E., Inui T., Lee T.W., Wachtler T., Sejnowski T.J., Spatio-chromatic receptive field properties derived from information-theoretic analyses of cone mosaic response to natural scenes. Neural Comp. 15, 2003, 397-417.

Field D.J., Relations between the statistics of natural images and the response properties of cortical cells. J. Opt. Soc. Am. A 4, 1987, 2379-2394.

Hoyer, P.O. and Hyvärinen A. Independent Component Analysis Applied to Feature Extraction from Colour and Stereo Images. Network: Computation in Neural Systems, 11, 2000, (3):191-210.

Hyvärinen, A., Oja, E. Independent Component Analysis: Algorithms and Applications. Neural Networks, 13, 2000, 411-430.

Hyvärinen, A. The FastICA package for Matlab. http://www.cis.hut.fi/projects/ica/fastica/, 2004.

Lee T., Wachtler T. and Sejnowski T.J., Color opponency is an efficient representation of spectral properties in natural scenes. Vis. Res. 42, 2002, 2095-2103.

Olshausen B.A., Field D.J., Emergence of simple-cell receptive field properties by learning a sparse code for natural images. Nature 381, 1996, 607-609.

Ruderman D.L., Cronin T.W., Chiao C.C., Statistics of cone responses to natural images: implication for visual coding. J. Opt. Soc. Am., 15, 1998, 2036-2045.

Tailor D.R, Finkel L.F., Buchsbaum G., Color-opponent receptive fields derived from independent component analysis of natural images. Vis. Res, 40, 2000, 2071-2076.

Wachtler T., Lee T.W., Sejnowski T.J., Chromatic structure of natural scenes. J. Opt. Soc. Am. A 18, 2001, 65-77. 